\title{
Predictive value of ACEF II score in patients with multi-vessel coronary artery disease undergoing one-stop hybrid coronary revascularization
}

Yanyan $\mathrm{Li}^{\dagger}$, Chuang Li ${ }^{\dagger}$, Dejing Feng, Qian Zhang, Kuibao Li, Yu Liu, Xinchun Yang and Lefeng Wang ${ }^{*}$

\begin{abstract}
Background: We aimed to investigate the predictive value of recently updated ACEF II score on major adverse cardiac and cerebrovascular events (MACCE) in patients with multi-vessel coronary artery disease (MVCAD) undergoing one-stop hybrid coronary revascularization (HCR).
\end{abstract}

Methods: Patients with MVCAD undergoing one-stop HCR were retrospectively recruited from March 2018 to September 2020. Several prediction risk models, including ACEF II score, were calculated for each patient. Kaplan-Meier curve was used to evaluate freedom from cardiac death and MACCE survival rates. Differences of prediction performance among risk scores for predicting MACCE were compared by receiver operating characteristic (ROC) curve.

Results: According to the ACEF II score, a total of 120 patients undergoing one-stop HCR were assigned to low-score group (80 cases) and high-score group (40 cases). During the median follow-up time of 18 months, the incidence of MACCE in the low-score group and high-score group were $8.8 \%$ and $37.5 \%$, respectively $(p<0.001)$; and the cardiac death rate of the two were $2.5 \%$ and $12.5 \%$, respectively $(p<0.05)$. Moreover, the cumulative freedom from cardiac death $(97.5 \%$ vs. $86.8, p<0.05)$ and MACCE $(75.2 \%$ vs. $52.8 \%, p<0.001)$ survival rates in the high-score group were significantly lower than in the low-score group. According to the Cox proportional hazards regression, the ACEF II score was an independent prognostic indicator for MACCE with hazards ratio (HR) 2.24, $p=0.003$. The ROC curve analysis indicated that the areas under the curve (AUC) of MACCE from the ACEF II score was $0.740(p<0.001)$, while the AUC of MACCE from the SYNTAX score II CABG was $0.621(p=0.070)$ and the AUC from the EuroSCORE II was 0.703 $(p<0.001)$. Thus, the accurate predictive value of ACEF II score was similar to the EuroSCORE II but much higher than the SYNTAX score II CABG.

Conclusions: The updated ACEF II score is a more convenient and validated prediction tool for MACCE in patients with MVCAD undergoing one-stop HCR comparing to other risk models.

Keywords: ACEF II score, One-stop hybrid coronary revascularization, Major adverse cardiac and cerebrovascular events, Multi-vessel coronary artery disease

*Correspondence: wlf368@163.com

†Yanyan Li and Chuang Li contributed equally to the work and should be regarded as co first authors

Heart Center and Beijing Key Laboratory of Hypertension, Beijing Chaoyang Hospital, Capital Medical University, No. 8 Gongti South Road, Beijing 100020, China

\section{Background}

Hybrid coronary revascularization (HCR), first proposed in 1996, is based on coronary artery bypass grafting $(\mathrm{CABG})$ by grafting the left internal mammary artery (LIMA) to left anterior descending artery (LAD) while 
performing percutaneous coronary intervention (PCI) on non-LAD vessels [1]. By implementing minimal access, HCR actually lowers the incidence of peri-operative complications, such as infection and transfusion, which often complicate CABG [2, 3]. Compared to multi-vessel PCI-stenting, the employment of LIMA graft reduces the number of stents required, decreases the risk of stents restenosis and thrombosis, and further improves the long-term survival rate $[4,5]$. However, in a prospective randomized trial, there were no significant difference between HCR and CABG in terms of the 1-year and 5-year rates of myocardial infarction, repeat revascularization, stroke or death $[6,7]$. And the incidence of mid-term major adverse cardiac and cerebrovascular events (MACCE) remained up to $20-25 \%$ for patients after HCR $[8,9]$. Thus, early and accurate identification of MVCAD patients who undergo HCR at high risk of MACCE is critical.

Currently, several risk models have been established and in use for predicting adverse events after PCI and CABG, such as EuroSCORE and SYNTAX score. The age, creatinine and ejection fraction (ACEF) score is a convenient and effective risk model for predicting in-hospital mortality in patients undergoing elective cardiac surgery, with an accurate predictive power comparable to that of EuroSCORE $[10,11]$. Subsequently, two variables including emergency surgery and pre-operative anemia were added into ACEF risk model, resulting in the updated ACEF II score. The ACEF II score has proven to be superior to the original model in discriminating and calibrating adverse outcomes in a large external area of cardiac surgery [12]. Though risk models could play a critical role in identifying high-risk patients following HCR, there is few evidence of which models are good predictors of poor outcomes. Hence, the objective of this study was to identify the best predictor for MACCE in patients with MVCAD referring for simultaneous HCR among current established risk models.

\section{Methods}

\section{Patient population}

A single-center and retrospective study was conducted at Beijing Chaoyang Hospital. Patients with angiographically confirmed MVCAD (stenosis $>50 \%$ of the lumen diameter in at least two major coronary arteries that involving the LAD) who underwent one-stop HCR (CABG first then followed PCI in the hybrid operating room) were consecutively enrolled from March 2018 to September 2020. Exclusion criteria included: (1) underwent staged HCR but not one-stop HCR; (2) life expectancy less than 1 year due to severe concomitant non-cardiac diseases, such as malignant tumor or significant infection; (3) incomplete data for calculating
ACEF II score; (4) contraindication to double antiplatelet therapy or drug-eluting stents; (5) lost during followup. Finally, 120 patients were enrolled into the analysis. The feasibility of one-stop HCR was at the discretion of the cardiac surgeons and interventionalists based on the results of coronary angiography and clinical characters at admission.

This study was approved by the institutional review board of Beijing Chaoyang Hospital and performed in accordance with the ethical standards laid down in the 1964 Declaration of Helsinki and its later amendments. All eligible patients had given written informed consent.

\section{Revascularization and pharmacological treatment}

The approach for HCR stages, surgical grafting of the LIMA-LAD was performed without the assistance of cardiopulmonary bypass and through a ministernotomy access. After closure of the thorax, angiography of the LIMA-LAD graft by femoral artery was immediately performed to confirm the patency. Then PCI was performed for remaining lesions. For the antiplatelet schemes, Aspirin $100 \mathrm{mg} /$ day was continued perioperatively, while clopidogrel was interrupted at least 7 days before the operation. Loading dose of clopidogrel $300 \mathrm{mg}$ was given after confirmation of LIMA-LAD graft patency. And the patients received unfractionated heparin (70-100 IU/kg body weight) intravenously prior to stenting, to achieve an activated clotting time of $>250$ s routinely. An early administration of aspirin $100 \mathrm{mg} /$ day and clopidogrel 75/ $\mathrm{mg}$ was performed on the first postoperative day. Then the dual antiplatelet therapy was maintained for 1 year and aspirin $100 \mathrm{mg} /$ day was administered indefinitely.

\section{Data collection and risk scores calculation}

The clinical and laboratory variables were collected from electronic medical records, such as age, sex, body mass index, left ventricular fraction (LVEF), haematocrit $(\mathrm{HCT})$, white blood cell, brain natriuretic peptide and serum creatinine.

The ACEF II score was calculated according to Ranucci $\mathrm{M}$ et al. using the following formula: ACEF II score $=$ Age $/ \mathrm{LVEF}+2.0$ (if serum creatinine $>2.0 \mathrm{mg} /$ $\mathrm{dL})+3.0$ (if emergency surgery) $+0.2 \times \mathrm{HCT}$ points below $36 \%$ [12], where the age was defined as completed years of the patients; the LVEF was defined as the percentage (\%) at the closest pre-procedural assessment; serum creatinine $(\mathrm{mg} / \mathrm{dL})$ was the last recorded just before surgery. Due to the high technique difficulty and the requirement for close operators cooperation, it was uncommon to perform one-stop HCR in an emergency situation, thus urgent HCR was considered as a variable instead of emergency surgery (urgent surgery was defined as the EuroSCORE II). The assessment of 
pre-operative anaemia was based on the last HCT value before operation.

SYNTAX score I-II was based on the assessment of angiographic features by two professional interventionalists. Coronary artery disease is defined as a narrowing of more than $50 \%$ of the lumen diameter in any major coronary arteries. SYNTAX score I-II was calculated on the basis of downloaded version from www.syntaxscore. com. For EuroSCORE I-II, pre-operative risk assessments were carried out for all patients by using the EuroSCORE systems. EuroSCORE I-II was evaluated according to the downloaded version from www.euroscore.pil-media.com.

\section{Clinical outcomes}

The median follow-up time of the present study was 18 months after discharge. All patients were regularly reviewed via either telephone or outpatient interviews. Besides, hospital documents and outpatient clinic interviews for MACCE were collected as well. The study endpoints were the composite endpoints of MACCE, including cardiac death, re-hospitalization for myocardial infarction, repeated revascularization and stroke. And the composite endpoint was assessed by time to first event.

\section{Statistical analysis}

SPSS (IBM, USA, version 25) and MedCalc (Seoul, Korea, version 19) were used for statistical analysis. Continuous variables were expressed as mean \pm standard deviation $(\mathrm{M} \pm \mathrm{SD})$ or median (interquartile range) in case of skewed distribution. Difference among groups was analyzed by Student's t test or Mann-Whitney U test. Categorical variables were presented as percentages (\%) and their statistical analysis were performed by the Chisquare test or Fisher's exact test. Cox proportional hazards model analysis was used to determine the potential risk factors for MACCE and the results were presented as hazards ratio (HR) and $95 \%$ confidential interval (CI). Variables with $p<0.1$ were included into the multivariate model for further analysis, except EuroSCORE, EuroSCORE II, SYNTAX score II CABG and the variables incorporating into the ACEF II score. Discrimination performance of ACEF II score and other risk scores for MACCE was accessed by receiver operating characteristic (ROC) curve analysis, and their areas under the curve (AUC) were compared using a nonparametric approach. Kaplan-Meier curve with Log rank test was applied to detect difference in event-free survival rates between two groups. The ROC curve analysis was used to determine the optimal cutoff value of the ACEF II score (1.35, sensitivity: $68 \%$, specificity: $75 \%$ ). All statistical tests were two-sided and variables with $p<0.05$ were considered statistically significant.

\section{Results}

\section{Baseline characteristics}

A total of 120 MVCAD patients who underwent onestop HCR were recruited and divided into two groups according to the cutoff value of ACEF II score, 80 cases in low-score group (ACEF II score $\leq 1.35$ ) and 40 cases in high-score group (ACEF II score $>1.35$ ). The baseline demographic characteristics were presented in Table 1. Significant difference was observed among age, NYHA class, brain natriuretic peptide, urgent operation, transfusion, coronary intensive care unit-time, complete revascularization, SYNTAX score II CABG, EuroSCORE and EuroSCORE II between the two groups. Moreover, the incidence of diabetes mellitus in the low-score group and the high-score group were $35.0 \%$ and $57.5 \%$, respectively, suggesting a significant difference between the two groups with $p<0.001$. Further analysis also indicated the HCT and LVEF were significantly lower in the high-score group.

\section{MACCE characteristics between two groups}

A total of 22 cases of MACCE (18.3\%) occurred during follow-up period. Compared to the occurrence of MACCE and cardiac death in the low-score group, with $8.8 \%$ and $2.5 \%$, respectively, they were significant higher in the high-score group, with $37.5 \%$ and $12.5 \%$, respectively (Table 2). While no significant difference was observed between the two groups regrading to the re-hospitalization for myocardial infarction, revascularization and stroke, their frequencies were higher in the high-score group.

\section{Freedom from MACCE and cardiac death survival rates between two groups}

The Kaplan-Meier curve indicated that the cumulative freedom from MACCE survival rate was significantly lower in the high-score group than in the low-score group $(75.2 \%$ vs. $52.8 \%, \log$ rank $=17.15, p<0.001)$ (Fig. 1a). And patients in the high-score group had lower freedom from cardiac death survival rate than those in the low-score group ( $97.5 \%$ vs. $86.8 \%, \log$ rank $=5.33$, $p=0.021$ ) (Fig. 1b). In addition, about $50 \%$ of patients suffered MACCE within 3-month after HCR.

\section{Cox proportional hazards model analysis of risk factors for MACCE}

The univariate Cox proportional hazards model analysis indicated that elevated ACEF II score was correlated with increased risk of MACCE (HR 2.60, 95\% CI 2.41$2.77, p<0.001)$, as well as diabetes mellitus, peripheral vascular disease (PVD), lymphocyte, HCT, EuroSCORE and EuroSCORE II $(p<0.05)$, as shown in Table 3. After multivariate adjustment, ACEF II score (HR 2.24, 95\% CI 
Table 1 Baseline demographics and clinical characteristics

\begin{tabular}{|c|c|c|c|}
\hline Characteristics & $\begin{array}{l}\text { Low-score group } \\
(n=80)\end{array}$ & $\begin{array}{l}\text { High-score group } \\
(n=40)\end{array}$ & $p$-value \\
\hline Age (years) & $62.3 \pm 9.8$ & $69.1 \pm 7.1$ & $<0.001$ \\
\hline Male, n (\%) & $67(83.8)$ & $32(80.0)$ & 0.610 \\
\hline Body mass index (kg/m2) & $26.6 \pm 2.8$ & $25.4 \pm 4.1$ & 0.065 \\
\hline \multicolumn{4}{|l|}{ Atadmission } \\
\hline Hypertension, n (\%) & $56(70.0)$ & $31(77.5)$ & 0.386 \\
\hline Diabetes mellitus, n (\%) & $28(35.0)$ & $23(57.5)$ & 0.019 \\
\hline Hyperlipidemia, n (\%) & $53(66.3)$ & $33(82.5)$ & 0.063 \\
\hline Smoking, n (\%) & $49(61.3)$ & $18(45.0)$ & 0.091 \\
\hline Cerebrovascular disease, n (\%) & $16(20.0)$ & $10(25.0)$ & 0.554 \\
\hline PVD, n (\%) & $14(17.5)$ & $10(25.0)$ & 0.333 \\
\hline Previous PCl, n (\%) & $16(20.0)$ & $14(35.0)$ & 0.074 \\
\hline LVEF (\%) & $66(62,70)$ & $55(48,63)$ & 0.001 \\
\hline \multicolumn{4}{|l|}{ NYHA class, n (\%) } \\
\hline$|-| \mid$ & $62(77.5)$ & $22(55.0)$ & 0.011 \\
\hline III-IV & $18(22.5)$ & $18(45.0)$ & - \\
\hline \multicolumn{4}{|l|}{ Clinical presentation, n (\%) } \\
\hline Stable coronary artery disease & $22(27.5)$ & $9(22.5)$ & 0.555 \\
\hline Unstable angina & $46(57.5)$ & $19(47.5)$ & 0.300 \\
\hline NSTEMI & $9(11.3)$ & $7(17.5)$ & 0.342 \\
\hline STEMI & $3(3.8)$ & $5(12.5)$ & 0.115 \\
\hline \multicolumn{4}{|l|}{ Culprit artery } \\
\hline LM, n (\%) & $30(37.5)$ & $16(40.0)$ & 0.564 \\
\hline LAD, n (\%) & $80(100.0)$ & $40(100.0)$ & - \\
\hline LCX, n (\%) & $50(62.5)$ & $30(75.0)$ & 0.357 \\
\hline RCA, n (\%) & $63(78.8)$ & $25(62.5)$ & 0.245 \\
\hline \multicolumn{4}{|l|}{ Laboratory assessment } \\
\hline $\mathrm{TC}(\mathrm{mmol} / \mathrm{L})$ & $3.6(3.1,4.5)$ & $3.7(3.1,4.1)$ & 0.628 \\
\hline TG $(\mathrm{mmol} / \mathrm{L})$ & $1.4(1.1,1.9)$ & $1.2(0.9,1.7)$ & 0.303 \\
\hline LDL-C (mmol/L) & $2.2(1.6,2.7)$ & $2.0(1.6,2.5)$ & 0.215 \\
\hline $\mathrm{HbA1c}(\%)$ & $6.3(5.8,6.9)$ & $6.2(5.9,7.7)$ & 0.557 \\
\hline Serum creatinine (mg/dL) & $0.79(0.69,0.92)$ & $0.80(0.72,1.03)$ & 0.207 \\
\hline $\mathrm{BNP}(\mathrm{pg} / \mathrm{mL})$ & $39.5(24.0,92.8)$ & $92.5(46.0,294.8)$ & $<0.001$ \\
\hline CK-MB (U/L) & $1.00(0.80,1.63)$ & $1.30(0.60,2.25)$ & 0.231 \\
\hline Tnl (ng/mL) & $0.01(0,0.04)$ & $0.02(0,0.71)$ & 0.072 \\
\hline WBC $(\times 10 \wedge 9 / L)$ & $6.8 \pm 1.6$ & $7.5 \pm 1.6$ & 0.330 \\
\hline Neutrophil (×10^9/L) & $4.2 \pm 1.4$ & $5.1 \pm 1.4$ & 0.145 \\
\hline Lymphocyte (×10^9/L) & $2.4 \pm 4.0$ & $1.7 \pm 0.51$ & 0.263 \\
\hline HCT (\%) & $40.7 \pm 3.1$ & $34.7 \pm 3.9$ & $<0.001$ \\
\hline \multicolumn{4}{|l|}{ Peri-operation } \\
\hline Urgent operation, n (\%) & $8(11.2)$ & $13(32.5)$ & 0.005 \\
\hline Elective operation, n (\%) & $71(88.8)$ & $27(67.5)$ & - \\
\hline Transfusion, n (\%) & $8(10.0)$ & $12(30.0)$ & 0.006 \\
\hline Reoperation, n (\%) & $2(2.5)$ & $3(7.5)$ & 0.196 \\
\hline Infection, n (\%) & $2(2.5)$ & $3(7.5)$ & 0.196 \\
\hline CCU-time (days) & $4(3,6)$ & $8(3,10)$ & 0.025 \\
\hline Complete revascularization, n (\%) & $65(81.3)$ & $25(62.5)$ & 0.025 \\
\hline \multicolumn{4}{|l|}{ Riskmodels } \\
\hline ACEF II score & $0.96 \pm 0.19$ & $6.53 \pm 2.55$ & $<0.001$ \\
\hline SYNTAX Score & $34.6 \pm 6.7$ & $33.9 \pm 6.9$ & 0.648 \\
\hline
\end{tabular}


Table 1 (continued)

\begin{tabular}{llll}
\hline Characteristics & $\begin{array}{l}\text { Low-score group } \\
(\mathbf{n = 8 0 )}\end{array}$ & $\begin{array}{l}\text { High-score group } \\
(\mathbf{n = 4 0 )}\end{array}$ & $\boldsymbol{p}$-value \\
\hline SYNTAX Score II CABG & $25.4 \pm 9.2$ & $32.4 \pm 9.0$ & $<0.001$ \\
EuroSCORE & $7.1 \pm 1.8$ & $8.7 \pm 1.8$ & $<0.001$ \\
EuroSCORE II & $2.4 \pm 1.6$ & $4.9 \pm 3.6$ & $<0.001$
\end{tabular}

Low-score group, ACEF II score $\leq 1.35$; High-score group, ACEF II score > 1.35

PVD, peripheral vascular disease; PCI, percutaneous coronary intervention; LVEF, left ventricular ejection fraction; NYHA, New York Heart Association; NSTEMI, nonST-segment elevation myocardial infarction; STEMI, ST-segment elevation myocardial infarction; LM, left main artery; LAD, left anterior descending artery; LCX, left circumflex artery; RCA, right coronary artery; TC, total cholesterol; TG, triglyceride; LDL-C, low-density lipoprotein cholesterol; HbA1c, glycated hemoglobin; BNP, brain natriuretic peptide; CK-MB, creatine kinase isoenzymes; CTnl, cardiac troponin I; WBC, white blood cell; HCT, haematocrit; CCU, coronary intensive care unit

Table 2 MACCE characteristics between two groups

\begin{tabular}{lcccc}
\hline Variables & $\begin{array}{l}\text { Total } \\
(\mathbf{n}=\mathbf{1 2 0})\end{array}$ & Low-score group $(\mathbf{n}=\mathbf{8 0})$ & High-score group $(\mathbf{n}=\mathbf{4 0})$ & $\boldsymbol{p}$-value \\
\hline MACCE, $\mathrm{n}(\%)$ & $22(18.3)$ & $7(8.8)$ & $15(37.5)$ & $<0.001$ \\
Cardiac death, $\mathrm{n}(\%)$ & $7(5.8)$ & $2(2.5)$ & $5(12.5)$ & 0.040 \\
Re-hospitalization for Ml, n (\%) & $5(4.2)$ & $2(2.5)$ & $3(7.5)$ & 0.332 \\
Revascularization, $\mathrm{n}(\%)$ & $6(5.0)$ & $2(2.5)$ & $4(10)$ & $3(7.5)$ \\
Stroke, $\mathrm{n}(\%)$ & $4(3.3)$ & $1(1.3)$ & 0.094 \\
\hline
\end{tabular}

Low-score group, ACEF II score $\leq 1.35$; High-score group, ACEF II score > 1.35

MACCE, major adverse cardiac and cerebrovascular events; $\mathrm{Ml}$, myocardial infarction

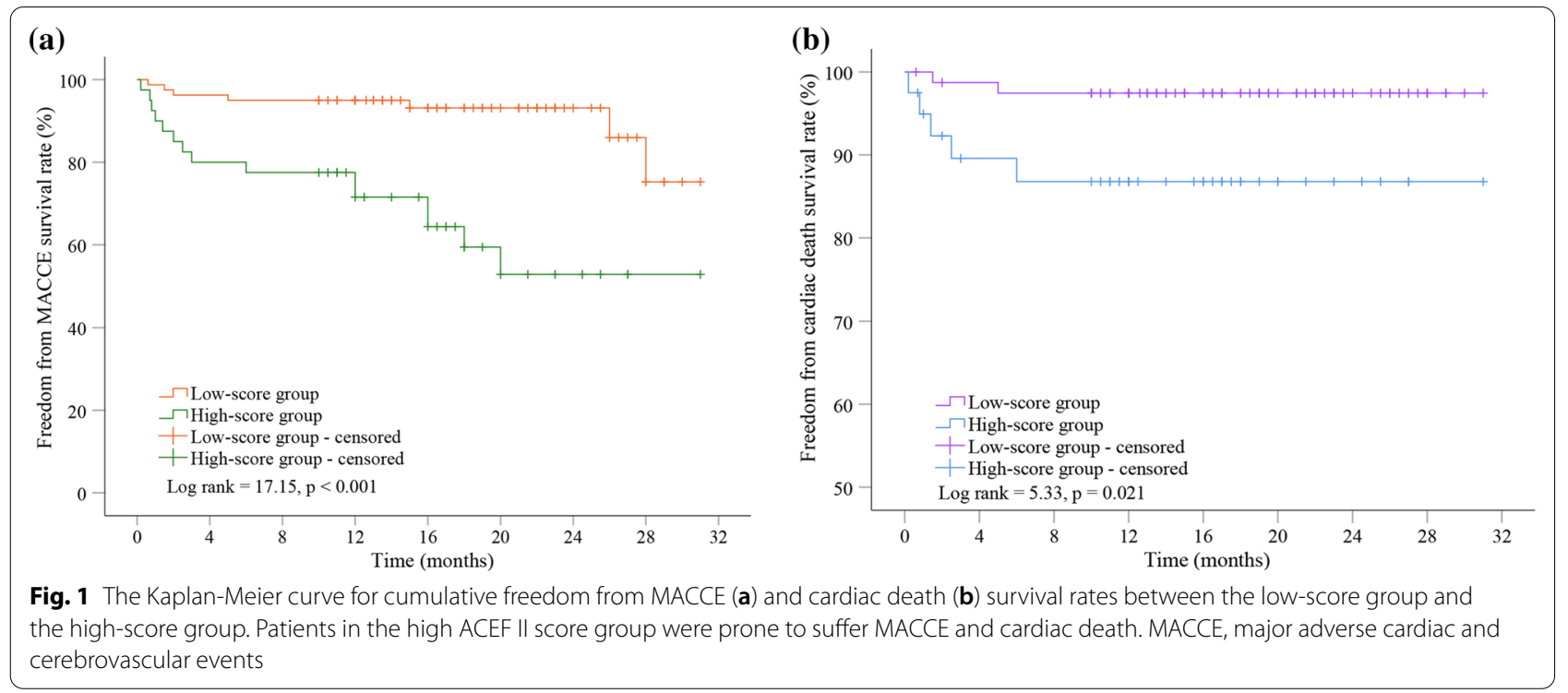

2.08-2.42, $p=0.003$ ) and diabetes mellitus (HR 3.23, 95\% CI $1.27-8.24, p=0.028)$ remained to be independent predictors for 18-month MACCE of HCR patients.

\section{Predictive values of the ACEF II score versus other risk scores for MACCE}

At ROC curve analysis, ACEF II score (AUC: 0.740, $p<0.001$ ), EuroSCORE (AUC: 0.671, $p=0.014$ ) and
EuroSCORE II (AUC: 0.703, $p<0.001$ ) presented similar excellent discrimination in predicting MACCE (as shown in Fig. 2). Meanwhile, SYNTAX score (AUC: 0.536, $p=0.590$ ) and SYNTAX score II CABG (AUC: 0.621, $p=0.070)$ had moderate discrimination in predicting MACCE. In addition, the ACEF II score had a sensitivity of $68.2 \%$ and specificity of $74.5 \%$ for predicting MACCE. When comparing ROC curves, the ACEF II score was 
Table 3 Univariate and multivariate analysis of MACCE

\begin{tabular}{|c|c|c|c|c|}
\hline \multirow[t]{2}{*}{ Variable } & \multicolumn{2}{|c|}{ Univariate analysis } & \multicolumn{2}{|c|}{ Multivariate analysis } \\
\hline & HR $(95 \% \mathrm{Cl})$ & $p$-value & HR $(95 \% \mathrm{Cl})$ & $p$-value \\
\hline Male & $1.33(0.44-3.98)$ & 0.612 & & \\
\hline Age & $1.03(0.99-1.08)$ & 0.159 & & \\
\hline Body mass index & $0.90(0.79-1.03)$ & 0.114 & & \\
\hline Hypertention & $1.29(0.48-3.51)$ & 0.611 & & \\
\hline Diabetes mellitus & $3.18(1.29-7.81)$ & 0.012 & $3.23(1.27-8.24)$ & 0.028 \\
\hline Hyperlipdemia & $1.15(0.45-2.97)$ & 0.770 & & \\
\hline Smoking & $0.89(0.38-2.06)$ & 0.777 & & \\
\hline Cerebrovascular disease & $1.85(0.75-4.58)$ & 0.181 & & \\
\hline PVD & $2.58(1.03-6.47)$ & 0.044 & $2.47(0.89-6.89)$ & 0.083 \\
\hline Previous PCl & $1.02(0.37-2.76)$ & 0.973 & & \\
\hline LVEF & $0.97(0.93-1.01)$ & 0.138 & & \\
\hline NYHA class & $1.37(0.57-3.32)$ & 0.485 & & \\
\hline Serum creatinine & $1.00(0.99-1.02)$ & 0.737 & & \\
\hline BNP & $1.00(0.99-1.01)$ & 0.204 & & \\
\hline CK-MB & $0.93(0.77-1.12)$ & 0.439 & & \\
\hline$c T n l$ & $0.99(0.97-1.02)$ & 0.745 & & \\
\hline WBC & $0.86(0.65-1.13)$ & 0.273 & & \\
\hline Neutrophil & $1.02(0.75-1.38)$ & 0.914 & & \\
\hline Lymphocyte & $0.30(0.13-0.70)$ & 0.006 & $0.67(0.24-1.85)$ & 0.435 \\
\hline $\mathrm{HCT}$ & $0.85(0.77-0.94)$ & 0.002 & & \\
\hline Transfusion & $1.56(0.57-4.22)$ & 0.385 & & \\
\hline Urgent operation & $1.72(0.23-12.96)$ & 0.600 & & \\
\hline CCU-time & $1.02(1.00-1.05)$ & 0.081 & $1.02(0.97-1.06)$ & 0.474 \\
\hline Complete revascularization & $0.95(0.37-2.44)$ & 0.916 & & \\
\hline ACEF II score & $2.60(2.41-2.77)$ & $<0.001$ & $2.24(2.08-2.42)$ & 0.003 \\
\hline EuroSCORE & $1.48(1.19-1.84)$ & $<0.001$ & & \\
\hline EuroSCORE II & $1.27(1.13-1.44)$ & $<0.001$ & & \\
\hline SYNTAX score & $0.97(0.92-1.02)$ & 0.291 & & \\
\hline SYNTAX score II CABG & $1.05(1.00-1.09)$ & 0.055 & & \\
\hline
\end{tabular}

MACCE, major adverse cardiac and cerebrovascular events; PVD, peripheral vascular disease; PCI, percutaneous coronary intervention; LVEF, left ventricular ejection fraction; NYHA, New York Heart Association; BNP, brain natriuretic peptide; CK-MB, creatine kinase isoenzyme; cTnl, cardiac troponin I; WBC, white blood cell; HCT, haematocrit; $\mathrm{CCU}$, coronary intensive care unit

a more accurate predictor than both EuroSCORE $[\Delta$ AUC: $0.069, p=0.333]$ and EuroSCORE II $[\Delta$ AUC: $0.037, p=0.555]$, although no statistical significance. Both the SYNTAX score [ $\triangle$ AUC: $0.204, p=0.013]$ and SYNTAX score II CABG [ $\triangle$ AUC: $0.119, p=0.042$ ] were significantly lower than the ACEF II score in predicting MACCE (Table 4).

\section{Discussion}

In the present study, we found that: (1) some MVCAD patients receiving one-stop HCR were still exposed to the high risk of cardiac death and MACCE; (2) the ACEF II score, which incorporates five clinical variables, was an independent risk factor for worse prognosis and had some potential for identifying patients at high risk of MACCE who underwent one-stop HCR; (3) similar to
EuroSCORE and EuroSCORE II, the ACEF II score presented excellent predictive power and was superior to both the SYNTAX score and SYNTAX score II CABG in predicting MACCE.

MVCAD is documented in $40-60 \%$ of coronary angiography patients and has a poorer prognosis compared with single-vessel disease [13]. The optimal extent of myocardial revascularization in patients with MVCAD is controversial and consensus is lacking. According to guideline recommendations, conventional CABG is considered as the standard technique for the management of MVCAD [14]. However, saphenous venous grafts to non-LAD targets are more inclined to progressive stenosis, with occlusion rates ranging from 6 to $30 \%$ as early as one year $[15,16]$. In contrast, the 12-month rates of stents restenosis and thrombosis after PCI are less than 


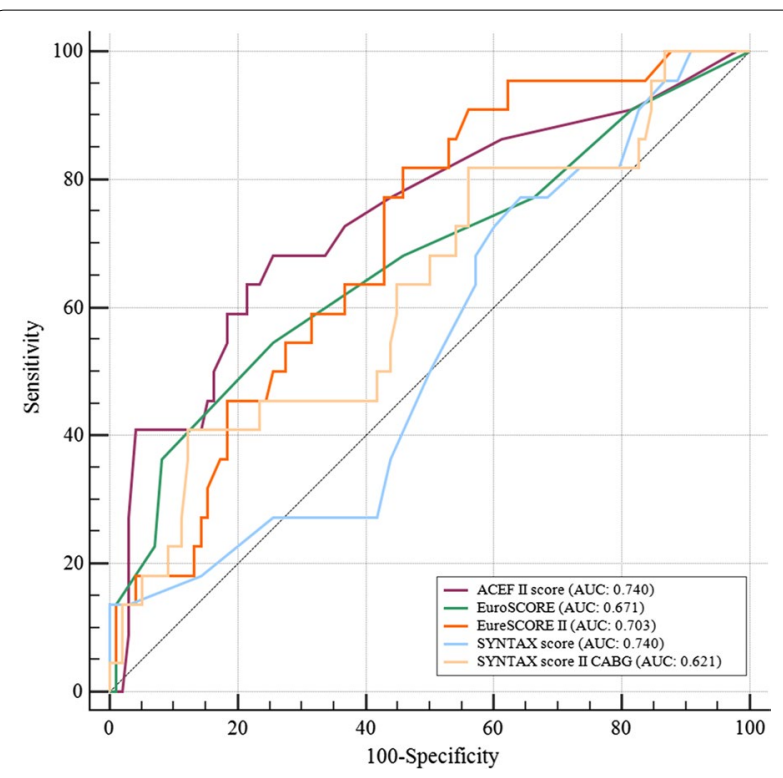

Fig. 2 Receiver operating characteristic (ROC) curve analysis of ACEF II score compared with other risk scores in predicting MACCE. AUC, areas under the curve

$5 \%$, especially after deployment of new-generation drugeluting stents [17]. Leveraging the advantages of both surgical and percutaneous techniques, HCR is expected to become a third coronary revascularization strategy for patients with MVCAD [18]. Moreover, the introduction of one-stop hybrid operating suites provides the opportunity for sequential surgical and percutaneous procedures, which can reduce the hospital stays and costs, also improve patients satisfaction $[19,20]$.

Notably, the high mortality and MACCE risk in MVCAD patients remains avoidable regardless of the optimized revascularization strategy [4, 21, 22]. The incidence of death and MACCE within 24 months after HCR was $5 \%$ and $19.3 \%$, respectively [23]. Consistent with prior research, we found cardiac death and MACCE rates after HCR were up to $5.8 \%$ and $18.3 \%$, respectively. Perhaps part reason of high risk of MACCE observed in one-stop HCR patients was the great complexity of coronary artery lesions, such as diffusion, calcification, bifurcation and chronic total occlusion. In such cases, PCI-stenting may be unable to achieve complete revascularization of non-LAD lesions, which is associated with a greater risk of short-term myocardial infarction, repeated revascularization and death [8]. According to the results of this study, the incidence of MACCE was predominantly driven by cardiac death (7 cases) and repeated revascularization (6 cases). Also, about $50 \%$ of patients developed MACCE within 3 months after HCR. On the other hand, the present study indicated that diabetes mellitus was also a robust predictor for adverse events following HCR. Patients with diabetes mellitus had more severe and diffuse atherosclerosis and aggressive pathological progression, resulting in the higher rates of coronary restenosis and new stenosis after coronary revascularization, which were strongly associated with the MACCE [20, 24]. Further, due to the diffuse nature of the atherosclerotic process, patients with MVCAD are often complicated with PVD. Previous studies have shown that PVD is an independent predictor of adverse outcomes and poorer survival after CABG and PCI [25, 26]. Although not statistically significant after multivariate adjustment analysis, there was still an association between PVD and poor prognosis. In conclusion, early risk stratification, management and therapy of MVCAD patients are essential before initiating HCR strategy.

The ACEF score, consisting of only a simple triple variable, has good predictive power for adverse events in cardiac surgery and PCI [10, 27]. By incorporated anemia and emergency surgery into the original model, Ranucci $\mathrm{M}$ et al. validated that the new ACEF II score provide superior discriminative power to the original score [12]. Though it was originally designed for cardiac surgery,

Table 4 Predictive value of ACEF II score versus other risk scores for MACCE

\begin{tabular}{|c|c|c|c|c|c|c|c|c|}
\hline & \multicolumn{3}{|c|}{ Single AUC analysis } & \multicolumn{5}{|c|}{ Difference between AUC } \\
\hline & AUC & $95 \% \mathrm{Cl}^{\mathrm{b}}$ & $p$-value & $\triangle \mathrm{AUC}$ & $95 \% \mathrm{Cl}^{\mathrm{b}}$ & $\mathrm{SE}^{\mathrm{a}}$ & Z statistic & $p$-value \\
\hline ACEF II score & 0.740 & $0.652-0.816$ & $<0.001$ & Reference & $\ldots$ & $\ldots$ & $\ldots$ & $\ldots$ \\
\hline EuroSCORE & 0.671 & $0.579-0.754$ & 0.014 & 0.069 & $(-0.071)-0.029$ & 0.071 & 0.967 & 0.333 \\
\hline EurOSCORE II & 0.703 & $0.613-0.783$ & $<0.001$ & 0.037 & $(-0.085)-0.519$ & 0.062 & 0.591 & 0.555 \\
\hline SYNTAX score & 0.536 & $0.442-0.627$ & 0.590 & 0.204 & $0.043-0.366$ & 0.082 & 2.477 & 0.013 \\
\hline SYNTAX score II CABG & 0.621 & $0.535-0.714$ & 0.070 & 0.119 & $(-0.031)-0.260$ & 0.072 & 1.853 & 0.042 \\
\hline
\end{tabular}

MACCE, major adverse cardiac and cerebrovascular events; AUC, areas under the curve

${ }^{a}$ Hanley and McNeil (1983)

${ }^{\mathrm{b}}$ Binomial exact 
ACEF II score also presented excellent predictive power in patients treated with primary PCI and in patients with aortic dissections undergoing interventional thoracic endovascular aortic repair surgery, demonstrating a significant correlation between elevated ACEF II score and increased risk of subsequent adverse events $[28,29]$. One-stop HCR is a simultaneous combination of surgical and interventional treatments including LIMA grafting to $\mathrm{LAD}$ and stents placement in other coronary lesions to manage MVCAD. Besides, previous studies have validated anemia and emergency surgery as prognostic markers for patients undergoing PCI or surgery $[30,31]$. Due to the similarity in surgical technique and management, we hypothesized that ACEF II score could be used as a reliable risk stratification model for one-stop HCR patients. As mentioned above, the present study demonstrated that ACEF II score has similar discriminatory power for predicting MACCE compared with EuroSCORE and EuroSCORE II [32]. Meanwhile, ACEF II score significantly outperformed both SYNTAX score and SYNTAX score II CABG in predicting MACCE [33]. Therefore, the ACEF II score is a relatively convenient and user-friendly model for pre-HCR risk stratification.

\section{Limitations}

Some limitations should be taken into account. First, the relatively small number of patients and the fact that the study was conducted in a single-center means that the prognostic value of the ACEF II score needs to be further confirmed in a large scale multi-center study. Currently, one-stop HCR is relatively difficult to implement because of the technical difficulty associated with it and the high expertise required of the operators, which is the main reason for the small sample size involved. Second, since the patients with MVCAD who only underwent onestop HCR were recruited, it was difficult to validate our findings in patients with staged HCR. Third, because of missed follow-up angiographic imaging data on coronary artery disease progression after HCR, we were unable to further assess its impact on the endpoint events in this study. Fourth, due to the limitation of medical costs, the functional testing (noninvasive/invasive) of coronary lesions was not routinely used for patients, which might lessen the accuracy of the coronary disease diagnosis inevitably. Finally, the follow-up period was relatively short.

\section{Conclusion}

ACEF II score, a relatively simple model, had been proved to be an independent predictor of MACCE in patients undergoing one-stop HCR. The ACEF II score could be used as a convenient and effective tool to guide physicians and surgeons in classifying high-risk MVCAD patients before HCR, thus potentially facilitating better clinical decision-making and treatment management.

\section{Abbreviations}

ACEF: Age, creatinine, ejection fraction; MACCE: Major adverse cardiac and cerebrovascular events; MVCAD: Multi-vessel coronary artery disease; HCR: Hybrid coronary revascularization; ROC: Receiver operating characteristic; HR: Hazards ratio; AUC: Areas under the curve; SYNTAX: Synergy between percutaneous coronary intervention with taxus and cardiac surgery; EuroSCORE: European System for Cardiac Operative Risk Evaluation; CABG: Coronary artery bypass grafting; LIMA: Left internal mammary artery; LAD: Left anterior descending artery; $\mathrm{PCI}$ : Percutaneous coronary intervention; LVEF: Left ventricular ejection fraction; HCT: Haematocrit; Cl: Confidential interval; NYHA: New York Heart Association; PVD: Peripheral vascular disease; NSTEMI: Non-ST-segment elevation myocardial infarction; STEMI: ST-segment elevation myocardial infarction; LM: Left main artery; LAD: Left anterior descending artery; LCX: Left circumflex artery; RCA: Right coronary artery; TC: Total cholesterol; TG: Triglyceride; LDL-C: Low-density lipoprotein cholesterol; HbA1c: Glycated hemoglobin; BNP: Brain natriuretic peptide; CK-MB: Creatine kinase isoenzyme; cTnl: Cardiac troponin I; WBC: White blood cell; CCU: Coronary intensive care unit; MI: Myocardial infarction.

\section{Acknowledgements}

Not applicable.

\section{Authors' contributions}

YYL and CL were involved in the study conception and design, data collection, data analysis and drafting of the manuscript. DJF contributed to data collection and analysis. QZ and YL were involved in the data analysis. KBL and XCY provided material and technical support and commented on the manuscript draft. LFW was responsible for data interpretation, commented on the design of this study and performed critical review. All authors have read and approved the manuscript.

\section{Funding}

The work has been supported by the National Key R\&D Program of China (2016YFC1301100, 2016YFC1301102). It funded the collection of data and manuscript text revision.

\section{Availability of data and materials}

The datasets generated and analyzed during the current study are not publicly available due to the restrictions by the Beijing Chaoyang Hospital who is the data owner. The authors used this dataset under an agreement with the Beijing Chaoyang Hospital for the current study. If someone needs to access the data used in the study on reasonable requests please contact the corresponding author Lefeng Wang.

\section{Declarations}

Ethics approval and consent to participate

This study was approved by the institutional review board of Beijing Chaoyang Hospital and performed in accordance with the ethical standards laid down in the 1964 Declaration of Helsinki and its later amendments. All eligible patients had given written informed consent.

\section{Consent for publication \\ Not applicable.}

\section{Competing interests}

The authors declare that they have no competing interests.

Received: 17 February 2021 Accepted: 27 September 2021

Published online: 10 October 2021 


\section{References}

1. Angelini GD, Wilde P, Salerno TA, Bosco G, Calafiore AM. Integrated left small thoracotomy and angioplasty for multivessel coronary artery revascularisation. Lancet. 1996;347(9003):757-8

2. Patel NC, Hemli JM, Kim MC, Seetharam K, Pirelli L, Brinster DR, et al. Short- and intermediate-term outcomes of hybrid coronary revascularization for double-vessel disease. J Thorac Cardiovasc Surg. 2018;156(5):1799 $-807 . e 3$

3. Puskas JD, Pawale A, Sharma SK. Hybrid coronary revascularization: a new treatment paradigm for selected patients with multivessel coronary artery disease. JACC Cardiovasc Interv. 2014;7(11):1284-6.

4. Modrau IS, Nielsen PH, Nielsen DV, Christiansen EH, Hoffmann T, Parner ET, et al. Outcome of hybrid compared to conventional revascularization in multivessel coronary artery disease. Scand Cardiovasc J. 2020;54(6):376-82.

5. Davidavicius G, Praet FV, Mansour S, Casselman F, Bartunek J, Degrieck I, et al. Hybrid revascularization strategy: a pilot study on the association of robotically enhanced minimally invasive direct coronary artery bypass surgery and fractional-flow-reserve-guided percutaneous coronary intervention. Circulation. 2005;112Suppl 9:1317-22.

6. Gąsior M, Zembala MO, Tajstra M, Filipiak K, Gierlotka M, Hrapkowicz T, et al. Hybrid revascularization for multivessel coronary artery disease. JACC Cardiovasc Interv. 2014;7(11):1277-83.

7. Tajstra M, Hrapkowicz T, Hawranek M, Filipiak K, Gierlotka M, Zembala M, et al. Hybrid coronary revascularization in selected patients with multivessel disease: 5-year clinical outcomes of the prospective randomized pilot study. JACC Cardiovasc Interv. 2018;11(9):847-52.

8. Modrau IS, Holm NR, Mæng M, Bøtker HE, Christiansen EH, Kristensen $\mathrm{SD}$, et al. One-year clinical and angiographic results of hybrid coronary revascularization. J Thorac Cardiovasc Surg. 2015;150(5):1181-6.

9. Bonatti JO, Zimrin D, Lehr EJ, Vesely M, Kon ZN, Wehman B, et al. Hybrid coronary revascularization using robotic totally endoscopic surgery: perioperative outcomes and 5-year results. Ann Thorac Surg. 2012;94(6):1920-6.

10. Ranucci M, Castelvecchio S, Menicanti L, Frigiola A, Pelissero G. Risk of assessing mortality risk in elective cardiac operations: age, creatinine, ejection fraction, and the law of parsimony. Circulation. 2009:119(24):3053-61.

11. Ranucci M, Castelvecchio S, Conte M, Megliola G, Speziale G, Fiore F, et al. The easier, the better: age, creatinine, ejection fraction score for operative mortality risk stratification in a series of 29,659 patients undergoing elective cardiac surgery. J Thorac Cardiovasc Surg. 2011;142(3):581-6.

12. Ranucci M, Pistuddi V, Scolletta S, de Vincentiis C, Menicanti L. The ACEF ॥ risk score for cardiac surgery: updated but still parsimonious. Eur Heart J. 2018;39(23):2183-9.

13. Bang WV, Levy MS. In multivessel coronary artery disease, a "state-of-theart" randomized clinical trial of revascularization is needed. Catheter Cardiovasc Interv. 2016;87(1):13-4.

14. Neumann FJ, Sousa-Uva M, Ahlsson A, Alfonso F, Banning AP, Benedetto $U$, et al. 2018 ESC/EACTS guidelines on myocardial revascularization. Eur Heart J. 2019;40(2):87-165

15. DesaiND, Naylor CD, Kiss A, Cohen EA, Feder Elituv R, Miwa S, et al. Impact ofpatient and target vessel characteristics on arterial and venous bypass graft patency:insight from a randomized trial. Circulation. 2007:115(6):684-91.

16. KimKB, Cho KR, Jeong DS. Midterm angiographic follow-up after offpump coronaryartery bypass: serial comparison using early, 1-year, and 5-year postoperativeangiograms. J Thorac Cardiovasc Surg. 2008;135(2):300-7.

17. Byrne RA, Joner M, Kastrati A. Stent thrombosis and restenosis: what have we learned and where are we going? The Andreas Grüntzig Lecture ESC 2014. Eur Heart J. 2015;36(47):3320-31.

18. Yanagawa B, Hong $K$, Cheema A, Bagai A, Verma S. What is the state of hybrid coronary revascularization in 2018? Curr Opin Cardiol. 2018;33(5):540-5.

19. Shen L, Hu S, Wang H, Xiong H, Zheng Z, Li L, et al. One-stop hybrid coronary revascularization versus coronary artery bypass grafting and percutaneous coronary intervention for the treatment of multivessel coronary artery disease: 3-year follow-up results from a single institution. J Am Coll Cardiol. 2013;61(25):2525-33.

20. Song Z, Shen L, Zheng Z, Xu B, Xiong H, Li L, et al. One-stop hybrid coronary revascularization versus off-pump coronary artery bypass in patients with diabetes mellitus. J Thorac Cardiovasc Surg. 2016;151(6):1695 - 701. e1.

21. Basman C, Hemli JM, Kim MC, Seetharam K, Brinster DR, Pirelli L, et al. Long-term survival in triple-vessel disease: hybrid coronary revascularization compared to contemporary revascularization methods. J Card Surg. 2020;35(10):2710-8.

22. Kitahara H, Hirai T, McCrorey M, Patel B, Nisivaco S, Nathan S, et al. Hybrid coronary revascularization: midterm outcomes of robotic multivessel bypass and percutaneous interventions. J Thorac Cardiovasc Surg. 2019;157(5):1829-36.e1.

23. Esteves V, Oliveira MAP, Feitosa FS, Mariani J Jr, Campos CM, Hajjar LA, et al. Late clinical outcomes of myocardial hybrid revascularization versus coronary artery bypass grafting for complex triple-vessel disease: long-term follow-up of the randomized MERGING clinical trial. Catheter Cardiovasc Interv. 2020.

24. Farkouh ME, Domanski M, Dangas GD, Godoy LC, Mack MJ, Siami FS, et al. Long-term survival following multivessel revascularization in patients with diabetes: the FREEDOM follow-on study. J Am Coll Cardiol. 2019;73(6):629-38.

25. O'Rourke DJ, Quinton HB, Piper W, Hernandez F, Morton J, Hettleman B, et al. Survival in patients with peripheral vascular disease after percutaneous coronary intervention and coronary artery bypass graft surgery. Ann Thorac Surg. 2004;78(2):466-70.

26. Ramzy J, Andrianopoulos N, Roberts L, Duffy SJ, Clark D, Teh AW, et al. Outcomes in patients with peripheral vascular disease following percutaneous coronary intervention. Catheter Cardiovasc Interv. 2019;94(4):588-97.

27. Gao S, Liu Q, Ding X, Chen H, Zhao X, Li H. Predictive value of the combination of age, creatinine, and ejection fraction score and diabetes in patients with ST-segment elevation myocardial infarction undergoing percutaneous coronary intervention. Coron Artery Dis. 2020;31(2):109-17.

28. Chichareon P, Modolo R, van Klaveren D, Takahashi K, Kogame N, Chang CC, et al. Predictive ability of ACEF and ACEF II score in patients undergoing percutaneous coronary intervention in the GLOBAL LEADERS study. Int J Cardiol. 2019;286:43-50.

29. Wei XB, Wang Y, Luo JF, Hong WZ, Su Z, Zhang CX, et al. Utility of age, creatinine, and ejection fraction score in patients with type $B$ aortic dissection undergoing thoracic endovascular aortic repair. Int J Cardiol. 2020;303:69-73.

30. Schumer EM, Chaney JH, Trivedi JR, Linsky PL, Williams M, Slaughter MS. Emergency coronary artery bypass grafting: indications and outcomes from 2003 through 2013. Tex Heart Inst J. 2016;43(3):214-9.

31. Pilgrim T, Vetterli F, Kalesan B, Stefanini GG, Räber L, Stortecky S, et al. The impact of anemia on long-term clinical outcome in patients undergoing revascularization with the unrestricted use of drug-eluting stents. Circ Cardiovasc Interv. 2012;5(2):202-10.

32. Musa AF, Cheong XP, Dillon J, Nordin RB. Validation of EuroSCORE II in patients undergoing coronary artery bypass grafting (CABG) surgery at the National Heart Institute, Kuala Lumpur: a retrospective review. F1000Res. 2018:7:534

33. He Y, Cheng XF, Wang K, Liu T, Fan HP, Pan WX, et al. Predictive value of combining the SYNTAX score with reactive hyperemia index in patients with acute coronary syndrome undergoing percutaneous coronary intervention. Catheter Cardiovasc Interv. 2020;95(5):E130-9.

\section{Publisher's Note}

Springer Nature remains neutral with regard to jurisdictional claims in published maps and institutional affiliations. 